\title{
O significado, as vivências e perspectivas de pacientes submetidos ao transplante renal
}

The meaning, experiences and perspectives of patients undergoing renal transplantation

El significado, las vivencias y perspectivas de pacientes sometidos al trasplante renal

Elaine Vanele Silvestre de Brito ${ }^{1}$, Maria da Conceição Baldez Duarte ${ }^{1}$, Fernanda Cardoso Rocha $^{1,2,3 *}$, Isabela Barbosa Cruz ${ }^{1}$, Gregório Ribeiro de Andrade Neto ${ }^{1,2}$, Géssica Pereira Barbosa ${ }^{1}$, Tharley Fabiano Silva Teixeira ${ }^{1}$, Ana Paula Oliveira Nascimento Alves ${ }^{1}$, Claudia Mendes Campos Versiani $^{1,2,3}$, Joyce Micaele Alves ${ }^{1}$, Meriele Santos Souza ${ }^{1}$, Leila das Graças Siqueira ${ }^{1,3}$.

\section{RESUMO}

Objetivo: Entender a definição, as experiências e sentidos dos pacientes que foram realizaram o transplante renal. Métodos: Realizou-se uma pesquisa de natureza qualitativa utilizando um roteiro não estruturado. Participaram da pesquisa 09 pacientes que submeteram ao transplante renal no Hospital do Rim da cidade de Montes Claros/MG no ano de 2015. A Técnica do Discurso do Sujeito Coletivo foi o recurso adotado para a análise dos dados. Resultados: A pesquisa revelou que os transplantados iniciaram uma nova vida após transplante, pois, passaram a vivenciar um "bem-estar", crescimento emocional retomando parte da vida como era antes da doença. Além disso, para os transplantados houve a necessidade de encontrar novas formas e meios de viver diante das condições após o transplante, o que se transformou em um fato positivo, pois, permitiu uma maior autonomia aos mesmos. Conclusão: Esta pesquisa possibilitou a participação e expressão dos transplantados de rim sobre os aspectos e mudanças ocorridas em sua vida após o transplante.

Palavras-chave: Transplante de Rim, Qualidade de vida, Emoções, Insuficiência Renal Crônica.

\begin{abstract}
Objective: Understand the definition, experiences and meanings of patients who underwent kidney transplantation. Methods: A qualitative research was carried out using an unstructured script. The study included 09 patients who underwent kidney transplantation at the Rim Hospital in the city of Montes Claros/MG in the year 2015. The Technique of the Discourse of the Collective Subject was the resource used to analyze the data. Results: The study revealed that transplant patients started a new life after transplantation, since they began to experience a "well-being", emotional growth resuming part of life as it was before the disease. New ways and means of living in the conditions after transplantation, which became a positive fact, therefore, allowed a greater autonomy to them. Conclusion: This research made possible the participation and expression of kidney transplants on the aspects and changes that occurred in your life after transplantation.
\end{abstract}

Key words: Kidney transplantation, Quality of life, Emotions, Chronic Renal Insufficiency.

\footnotetext{
${ }^{1}$ Faculdades Unidas do Norte (FUNORTE), Montes Claros-MG. * E-mail: nandac.rocha@hotmail.com

${ }^{2}$ Faculdade de Saúde Ibituruna (FASI), Montes Claros-MG.

3 Universidade Estadual de Montes Claros (UNIMONTES), Montes Claros-MG.
} 


\section{RESUMEN}

Objetivo: Comprender el significado, las vivencias y perspectivas de pacientes que se sometieron al trasplante renal. Métodos: Se realizó una investigación de naturaleza cualitativa utilizando un guión no estructurado. En el año de 2015, la técnica del Discurso del Sujeto Colectivo fue el recurso adoptado para el análisis de los datos. Resultados: La investigación reveló que los trasplantados iniciaron una nueva vida tras trasplante, pues, pasaron a experimentar un "bienestar", crecimiento emocional retomando parte de la vida como era antes de la enfermedad. Además, para los trasplantados hubo la necesidad de encontrar y en el caso de que se produzca un cambio en la calidad de vida de las personas, en su vida después del trasplante lo que se transformó en un hecho positivo, pues, permitió una mayor autonomía a los mismos. Conclusión: Esta investigación posibilitó la participación y expresión de los trasplantes de riñón sobre los aspectos y cambios ocurridos en su vida después del trasplante.

Palabras clave: Trasplante de riñón, Calidad de vida, Emociones, Insuficiencia renal cronica.

\section{INTRODUÇÃO}

Define-se Insuficiência Renal Crônica (IRC) como sendo uma doença assintomática que pode acompanhar o indivíduo por muitos anos e só manifestar-se quando já existira perda de quase a totalidade do funcionamento dos rins, em torno de $80 \%$ de perda, sem condição de reversão do quadro, levando o doente à necessidade de um tratamento substitutivo do rim (BRASIL, 2014). Destaca-se ainda que a IRC é um problema mundial de saúde pública, pois, as doenças do rim e trato urinário são responsáveis por cerca de 850 mil mortes por ano e 15 milhões de anos de vida regulados por incapacidade, constituindo-se na $12^{\underline{a}}$ causa de morte e na $17^{\underline{a}}$ causa de incapacidade (CHERCHIGLIAI et al., 2010).

A IRC é singular pelo fato de dispor de três modalidades de terapia: Tratamento etiológico centrado no estabelecimento de dieta hipoproteica e uso de medicamentos; Tratamento dialítico onde substitui duas importantes funções renais: a remoção de solutos e a eliminação de líquido, porém, não substitui a função endócrina, e, portanto, não controla distúrbios como o hiper paratireoidismo secundário, a osteodistrofia e a anemia (CHERCHIGLIAI et al., 2010).

Tratamento hemodialítico que consiste na remoção de solutos e ocorre predominantemente por difusão, que se refere ao movimento de solutos do compartimento sanguíneo para o compartimento de dialisado, através de uma membrana semipermeável e a Transplantação do Rim que é uma intervenção cirúrgica no qual é aderido um enxerto renal ao recebedor, podendo ser de concessor que tenha vida ou de um defunto conciliável, sem contraindicações para doação (MATOS e LUGON, 2014; AZEVEDO et al., 2015; MARTINS e CESARINO, 2005).

Esse tipo de tratamento não tem caráter curativo, contudo busca aliviar os sintomas, conservando a vida, apesar de todas estarem disponíveis gratuitamente a todos os brasileiros através do Sistema Único de Saúde (SUS) (BRASIL, 2012). Mas, a abordagem ao tema que envolve transplante de órgãos é importante, pois, vem provocando grande repercussão no meio médico-científico, bem como a toda a população, de uma forma geral, sobretudo o transplante renal, por se tratar de aspectos condicionados como o avançar da medicina e da tecnologia, ademais incitando à sentimentos e emoções, correlacionados intimamente à representação da vida e da morte (BRASIL, 2014). Ravagnani et al., (2007) apontam os benefícios do transplante, mas também descrevem que o risco da rejeição do órgão, além da dúvida de sobreviver, prejudicam muito, aumentando diretamente o estado psicológico do paciente transplantado.

Entretanto, Fontoura (2012) afirma que as pessoas que realizaram o transplante do rim, conviveram com várias alterações renais que podem atuar diretamente na integralidade de vida do sujeito. A autora afirma ainda que o conhecimento e a prática que possuem não são suficientes para que eles continuem a levar uma vida saudável. Para o paciente portador de IRC, o transplante significa uma expectativa na qualidade de vida, sobretudo pela crença à libertação e independência da máquina de diálise como resultado a cura promovida 
pelos pós transplante, para que ações futuras possam ser planejadas para aumentar a qualidade de vida desses clientes.

Assim, esta pesquisa tem como objetivo compreender o significado, as vivências e perspectivas de pacientes submetidos ao transplante renal em um hospital na cidade de Montes Claros durante o ano de 2015.

\section{MÉTODOS}

Realizou-se uma pesquisa qualitativa do tipo descritiva com pacientes que foram submetidos a transplante renal no Hospital do Rim de Montes Claros/MG durante o ano de 2015. Participaram da pesquisa nove dos trinta e três pacientes que foram submetidos a transplante renal durante o ano de 2015. A identificação dos pacientes deu-se a partir dos endereços e contatos dos pacientes junto ao hospital. E, logo, após com a autorização do hospital do rim, houve a identificação dos participantes, sendo os mesmos contatados por telefone para agendar data e horário para a coleta dos dados que ocorreu somente junto aos pacientes que residiam na cidade de Montes Claros/MG.

Para a coleta de dados utilizou a técnica de entrevista por meio de um roteiro não estruturado contendo questões norteadoras sobre o tema pesquisado. Ressalta-se ainda, que as entrevistas foram gravadas somente após autorização do paciente e a coleta ocorreu no domicilio, considerando a privacidade e o conforto do paciente para responder às perguntas sem precisar tirá-lo da sua rotina diária. Assim, a coleta de dados por meio de entrevista permitiu que o transplantado emitisse opinião/sentimentos e comparações em relação ao pré e pós transplante renal e as mudanças ocorridas relacionadas à vida pessoal, familiar e social.

Destaca-se ainda que foi garantido o anonimato, bem como, foi solicitado aos mesmos a permissão para gravar as entrevistas e que os mesmos assinassem o termo de consentimento. E para preservar o anonimato dos entrevistados, os pacientes foram codificados pela letra $E$ de Entrevistada seguido de número arábico obedecendo à ordem de coleta dos dados. Quanto aos aspectos éticos e legais, a pesquisa foi realizada mediante autorização do Comitê de Ética em Pesquisa da Associação Educativa do Brasil - SOEBRAS conforme parecer consubstanciado ํㅜ 1.719.859de 10 de agosto de 2016 no intuito de atender às exigências do Conselho Nacional de Saúde, o qual aborda as diretrizes e normas regulamentadoras de estudo envolvendo seres humanos (BRASIL, 2012).

A análise dos resultados utilizou-se da técnica do discurso do sujeito, que é uma abordagem de construção do pensamento, com o intuito de desvelar o pensamento do sujeito, os sentidos e manifestações sobre determinado fenômeno, é uma coparticipação de ideias de um grupo. Assim, os dados foram agrupados pelas semelhanças de significados e apresentados nas seções resultados e discussão (LEFÈVRE e LEFĖVRE, 2005).

\section{RESULTADOS}

Os resultados deste estudo apontam para aspectos relacionados às questões norteadoras do estudo que foram utilizadas durante o desenvolvimento da pesquisa, onde pelos depoimentos, encontrou-se a interpretação, as experiências, sentidos e as alterações importantes realizadas na vida dos indivíduos que foram submetidos a transplante renal definindo assim as seguintes 04 categorias resultantes da pesquisa.

\section{$A$ vida antes do transplante renal}

Inicia-se a apresentação dos resultados chamando a atenção que a IRC e o processo de tratamento hemodialítico é considerado um processo que deixa marcas na vida das pessoas mesmo após se submeterem a transplantes renais, pois, para muitos, a hemodiálise é tida como um aprisionamento a máquina. E mesmo sendo um processo tão difícil, a hemodiálise garante maior sobrevida, podendo ter a esperança de almejar 
um transplante para melhorar sua qualidade de vida, pois o transplante é considerado,neste caso, uma esperança de vida melhor. Fato este que pode ser observado nas falas dos entrevistados abaixo:

"Só que eu fiquei nove anos na... na máquina e sempre aguardando e confiando em Deus e sempre esperando né?! Um transplante...” E2.

“...Vichi, sentia como uma presa, sem ter liberdade, tem sua vida, mas é muito limitada, limitada...” E3. E4.

"...é como se a gente se vive para fazer hemodiálise e não fizesse hemodiálise para viver que é o foco né ?!"

“...porque é muito complicado agente que tem o problema né, ter de ser submetido três vezes na semana o processo de hemodiálise...” E7.

“...era muito ruim, eu não podia beber água, não dormia direito, praticamente não tinha paz...” E9.

\section{O Significado do transplante renal}

Para os pacientes pesquisados o transplante tem significado de esperança e de fé, proporcionando à pessoa doente a oportunidade de libertar-se da dependência da hemodiálise, retomar sua autonomia de vida. $\mathrm{E}$ dentre os aspectos destacados que mais contribuem para tal significação, destacam-se o tempo e os sentimentos que vivenciaram durante o processo de hemodiálise e as mudanças ocorridas a partir do transplante.

“..."cê" tá livre, eu contava as horas de...é como um escravo que recebe a carta de alforria...” E3.

“... ficava quatro horas na máquina...e é para "mim" a morte; era morte, como ia fazer, deixar uma filha de apenas cinco anos... eu agradeço muito a Deus por isso, agora foi uma benção..." E2.

“... a pessoa tem acesso de "renascer de novo"... O transplante, isso não ocorre de um dia pro outro e nem em três dias, é muito tempo, só que eu, fiquei oito anos na máquina, muito tempo...” E1.

“...após o transplante você tem o direito a viver, alimentar normal, tomar água né, à vida da gente muda por completo eu me sinto como se eu tivesse ganhado uma nova vida." E7.

\section{Dificuldades enfrentadas após o transplante renal}

As falas abaixo permitem descrever que os pacientes transplantados demonstraram conhecimento e esclarecimento sobre a necessidade de realizar o acompanhamento clínico e a importância da realização de consultas e exames de rotinas após o transplante renal, relacionados por muitos, como uma dificuldade devido ao fato de lembrar sempre da doença e do medo de complicações do transplante.

“...faço tudo direitinho, tudo que é mandado...acompanhei todos os exames que eu fazia mensal lá, eu pegava uma cópia dos exames para mim..."E1.

“... depois do transplante nos primeiros meses a gente tem muita dificuldade de adaptação, a gente sente muita dor nos primeiros dias, a gente sente muito efeito colateral de tudo quanto é remédio que você toma...você fica com o emocional totalmente descontrolado por conta dos remédios... um nervoso sem explicação..." E4.

“...pois é o rim não é uma coisa... que vai durar para o resto da vida se foi transplantado nunca mais você vai ter esse problema...depende da gente e do cuidado." E1.

“.... sensação de corpo ruim nos primeiros seis meses...então eu acho assim que o organismo adaptou ao rim, mas no começo da adaptação foi difícil..." E4.

\section{Mudanças ocorridas após o transplante e o impacto na sua qualidade de vida}

Os significados e percepções que envolvem o transplante renal evidenciam que os pacientes atribuem um novo significado à essa nova vida que inicia a partir do transplante renal, pois, o tratamento hemodialítico, afeta de forma muito profunda as relações familiares que acabam modificando hábitos, em solidariedade e motivação, bem como, a própria vida. 
“...tudo transformou na minha vida, aí "tô" tomando o remédio direitinho "tô" fazendo tudo direitinho...E6.

“...eu não sinto mais problema de rins, eu vi que a minha pele está totalmente diferente, a saúde melhorou, e esse aspecto de voltar a viver normalmente, que é o mais importante né ?!” E4.

“...então minha vida mudou, eu "tô" me sentindo muito bem, eu viajo, antigamente não podia viajar.... Eu "tava" isolado, mas depois do transplante foi muito bom...E9.

“... foi muito importante pra gente, sempre estar com a família onde estiver, e então a preocupação é menos..."E2.

“... hoje eu "tô" convivendo mais com a família..." E3.

“.... então na família eu dei um descanso né?! Para os meus familiares E4.

“... na minha vida social teve poucas mudanças, e a familiar e o modo de viver sozinho, tive mas minha mãe e eu vivo com ela super bem, ela faz de tudo que eu posso comer, vivo a vida..." E5.

“.... agora posso tocar minha vida normal. E na minha vida familiar mudou muito também, que agora eu convivo muito com minha família..." E7.

“...mudou tudo porque quando a pessoas tá num tratamento desse não sofre só a pessoa, sofre a família também, meus filhos, meus amigos; graças a Deus a comunidade "mim" ajudou muito, e só de"cê" vê a felicidade das pessoas e participar do dia a dia da gente..." E8.

\section{Expectativas para o futuro}

Destaca-se que é produzida uma significação sobre o transplante do rim que é definida como expectativas para o futuro, conforme apresenta as falas abaixo:

“... depende você ter fé em Deus e o psicológico bom se não tiver não consegue e a perspectiva melhora..." E1. E4.

“... e poder conseguir fazer as coisas que eu queria fazer e não podia antes do transplante..."

"...hoje eu penso mais em viver e fazer minha filha feliz e minha família feliz que "pra" isso eu precisava..." E2.

"Começo mais sonhando, eu mesmo agora estou fazendo uns cursos...tenho vontade mais à frente fazer uma faculdade, tira carteira de carro, estou com uns projetos ver se Deus permite... e agora posso realizar esses sonhos que tenho vontade...E3.

"Olha, eu pretendo terminar o que comecei a fazer, pretendo trabalhar "pra"que eu possa conseguir algumas coisas que não "tava" podendo." E4.

\section{DISCUSSÃO}

\section{A vida antes do transplante}

A esperança da realização do transplante, ou o tempo de espera foi um período apresentado por todos os entrevistados como se fosse de muita espera, causando sentimentos de incerteza, tristeza e frustração, pois, para os mesmos, os dias passavam e eles permaneciam na expectativa, conforme foi descrito na presente pesquisa, onde os pacientes deixaram transparecer o significado de limitação e a compreensão de um mundo marcado por regras, horários e preocupações,pois, para eles o tratamento dialítico em muitos momentos provoca dor, revolta e tristeza. Este fato também foi evidenciado por Souza e Dutra (2013) e nos estudos de Santos et al., (2016)onde destacam que o transplante renal tem sido considerado há anos como um tratamento que proporciona melhor qualidade de vida às pessoas com IRC. A libertação do tratamento pelo diálise, apresenta vários significantes, dentre eles a liberdade de fazer coisas que devido o tratamento não podia fazer, como realizar passeios, alimentar-se do que deseja, aumentar a ingesta hídrica etc. O transplante é apresentando ao doente bem como a família, como uma dos melhores recursos para a terapêutica biopsicosocial e econômica, contudo, ressalta-se que não exclui o caráter crônico da condição do indivíduo, 
devendo ter cuidados rigorosos aos cuidados, bem como a ingestão adequada da medicação e o acompanhamento médico constante.

\section{O Significado do transplante renal}

Os achados ou depoimentos descritos no estudo desenvolvido por Fontoura (2012) permitem interpretar a noção do transplante como um novo começo, uma nova chance de viver vista como uma condição de livramento, contentamento, um segmento da vida e modificações de princípios; em suma, na forma de compreender a vida.

Silva et al., (2013) mostram que as expectativas e esperança em torno do transplante, volta-se pelo desejo de se ter uma vida nova, aliado a uma mudança significativa na qualidade de vida e sobretudo no desejo de recomeçar, com condições biopsicossocial propicio. Com isso a possibilidade do transplante, apresenta-se como realidade desde quando o doente é notificado sobre a necessidade do transplante, consequentemente a inclusão deste na "fila" de transplante sendo, assim eles, os doentes, compreende essa possibilidade como único recurso e maneira de retornar a suas condições de vida de antes da doença, entendendo esse transplante com uma possibilidade de cura e retorno à vida dita "normal" (KNIHS et al., 2013).

\section{Principais dificuldades enfrentadas após o transplante renal}

Destaca-se que os muitos benefícios relacionados para melhor qualidade de vida das pessoas com IRC estejam e são oriundos a partir da possibilidade do transplante renal, mas, ressalta-se que os indivíduos transplantados necessitam também estabelecer um estilo de vida diferenciado no que diz respeito à alimentação, higiene, medicamentos e cuidados com a saúde, bem como, os comportamentos relacionados às visitas regulares ao médico, o uso de medicamentos e melhor controle da dieta apresentando correlação positiva com a percepção de qualidade de vida (LIRA e LOPES, 2012).

Quintana e Hermann (2011); Lira e Lopes (2012) afirmam que o transplante proporciona a pessoa novo sentido a sua existência, encontrando novas formas e meios de viver diante das condições após o transplante. O paciente ainda vivencia o constante risco de rejeição do órgão enxertado, fazendo-se necessária a utilização diária de drogas imunossupressoras, e a adequação a um novo modo de vida.

Santos et al., (2016) afirmam que o transplante para o paciente possibilita restabelecimentos no estado biopsicosocial, agregando disposição para a realização de atividades rotineiras, antes impossibilitadas durante o tratamento.

Novamente, Fontoura (2012), lembra que o estado de saúde dos transplantados é visto de maneira subjetiva, assim, como as dificuldades, que variam de uma pessoa para outra, fato este que também foi evidenciado na presente pesquisa.

\section{Mudanças após o transplante e o impacto na qualidade de vida}

Nesta pesquisa pode-se perceber que apesar dos diferentes sentidos dispostos pelos pacientes a realização do transplante renal, a existência pós transplante acarretou um sentido de expectativa para uma vida melhor. A expectativa proveniente do transplante renal engloba as proporções socioculturais existentes na vida de cada pessoa, a ele submetido. Terra e Costa (2007) descrevem que o transplante renal, além de permitir a reabilitação do paciente a um estilo de vida normal, possibilita também uma economia significativa para o sistema de saúde. Cabe ressaltar que, embora o transplante tenha sido considerado o tratamento de melhor custo-efetividade deve-se considerar o contratempo da captação de órgãos e a necessidade de uma central de transplante bem equipada e uma equipe multidisciplinar definida e competente.

Ainda pelos estudos consultados de Santoset al., (2016), pode-se dizer que o transplante renal pode repercutir na vida social, acarretando melhora nas interações, além de prestar ações solidárias que auxiliem outras pessoas que sofrem com a IRC. 


\section{Expectativas para o futuro}

Fontoura (2012) relata que as chances readquiridas pela pessoa posteriormente ao transplante renal possibilitou aumentar a esperança entre os portadores de IRC se assegura após receber o rim que tanto desejava e os estudos de Cabral (2009) traz que a esperança é demonstrada como uma força interior promotora da vivência. Nesta pesquisa pode-se perceber que a esperança foi renovada após o transplante e que houve uma transformação, mas agora, benéfica, que os permitiu ter sensações de bem-estar, alegria, sonhos e liberdade.

Cria-se expectativas de mudanças, as pessoas transplantadas passam a acreditar e a vivenciar um "bemestar", crescimento emocional associado ao transplante e as mudanças relacionadas a sua qualidade de vida. Podendo retomar parte da sua vida como era antes de iniciar a hemodiálise.

Santos et al., (2016) afirmam que independentemente das diversas características positivas e negativas que o transplante produz ao paciente com IRC, constata-se que a realização desse procedimento possibilita um novo sentido de suas vidas, que eram marcadas pela realização de sessões de diálise por um longo período.Assim a expectativa é que a realização do transplante renal, está tão almejada a liberdade que vem para trazer muitos benefícios a suas vidas, como uma mudança brusca em sua rotina, sem a obrigação de sessões semanais de tratamento (Silva, 2015).

\section{CONCLUSÃO}

Este estudo proporcionou compreender que o transplante renal tem um significado de bênção para a pessoa com insuficiência renal crônica e representa a expectativa de poder retornar a sua vida saudável e continuar de onde foi interrompida, ou seja, representa a esperança de uma vida melhor e de poder voltar a sonhar com um futuro próspero, o que exigirá cuidados pessoais, dos familiares e de profissionais, caracterizando assim a rede de cuidados e apoio a pessoa transplantada.Conclui-se, que este trabalho colabora para revelar a visão e significação das vivências das pessoas transplantadas de rim sobre a importância do transplante em suas vidas.

\section{REFERÊNCIAS}

1. AZEVEDO SM, SIQUEIRA A, MANHÃES LSP. Insuficiência Renal Crônica: Análise do Binômio Enfermeiro Portador de IRC. Persp. Online:biol.\& saúde, 2015; 19(5):11-34.

2. BRASIL. Resolução n. 466, de 12 de dezembro de 2012. Brasília; O Plenário do Conselho Nacional de Saúde em sua 240 ${ }^{-}$Reunião Ordinária, realizada nos dias 11 e 12 de dezembro de 2012, no uso de suas competências regimentais e atribuições conferidas pela Lei no 8.080, de 19 de setembro de 1990, e pela Lei № 8.142, de 28 de dezembro de 1990. Diário Oficial da República Federativa do Brasil, Brasília, DF, 2012.

3. BRASIL. Ministério da Saúde. Secretaria de Atenção à Saúde. Departamento de Atenção Especializada e Temática. Diretrizes Clínicas para o Cuidado ao paciente com Doença Renal Crônica - DRC no Sistema Único de Saúde/ Ministério da Saúde. Secretaria de Atenção à Saúde. Departamento de Atenção Especializada e Temática. Brasília: Ministério da Saúde, 2014.

4. CABRAL HF. Idealizar o amanhã, vivendo entre a esperança e a desesperança num transplante renal. CuidArte Enfermagem, 2009; 3(2): 113-119.

5. CHERCHIGLIA ML, MACHADO EL, SZUSTER DAC et al. Perfil epidemiológico dos pacientes em terapia renal substitutiva no Brasil, 2000-2004. Revista de Saúde Pública, 2010; 44(4): 639-649.

6. FONTOURA FAP. A compreensão de vida de pacientes submetidos ao transplante renal: significados, vivências e qualidade de vida. Dissertação (Mestrado em Psicologia) - Universidade Católica Dom Bosco, Campo Grande, 2012.

7. LEFÈVRE F e LEFÈVRE AMC. O discurso do sujeito coletivo: um novo enfoque em pesquisa qualitativa (desdobramentos). 2. ed. Caxias do Sul: Educs, 2005.

8. KNIHS NS, SARTOR LD, ZINK D et al., A vivência de pacientes que necessitam de transplante renal na espera por um órgão compatível. Texto contexto enferm., 2013; 22(4): 1160-1168. 
9. LIRA ALBC e LOPES MVO.Pacientes Transplantados Renais: análise de associação dos diagnósticos de enfermagem. Revista Gaúcha de Enfermagem, 2010; 31(1):108-14.

10. MARTINS MRI e CESARINO CB. Qualidade de vida de pessoas com doença renal crônica em tratamento hemodialítico. Revista Latino-Americana de Enfermagem, 2005; 13(5): 670-676.

11. MATOS JPS e LUGON JR. Hora de conhecer a dimensão da doença renal crônico no Brasil. J.Bras. Nefrol, $2014 ; 36(3):$ 267-268.

12. QUINTANA e HERMANN WC. Atribuições de significados ao transplante renal. Psico [online], 2011; 42(1): 2330.

13. SANTOS BP dos, VIEGAS AC, GUANILO MEE et al. Situações vivenciadas após o transplante de rim; Saúde Ver, 2016; 16(42): 71-81.

14. SILVA LC, FREITAS TS, MARUYAMA SAT et al.O Transplante Renal na Perspectiva da Pessoa Transplantada, CiencCuid Saude,2013;12(2): 356-364.

15. SILVA JdeS.Representações Sociais de Clientes em Hemodiálise e Familiares Sobre o Transplante Renal. Dissertação (Mestrado em Enfermagem e Saúde) - Universidade Estadual do Sudoeste da Bahia. Jequié, 2015.

16. SOUSA AMD e DUTRA LMA.Qualidade de Vida dos Pacientes Submetidos a Transplante Renal. Com. Ciências Saúde. 2013; 24(1): 9-20.

17. TAVARES E. A vida depois da vida: reabilitação psicológica e social na transplantação de órgãos. Análise Psicológica, 2004; 22(4):765-777.

18. TERRA F de S e COSTA AMDD. Expectativa de vida e renais crônicos. R Enferm UERJ, 2007;15(4): 533-537. 